\title{
2245. Implementation of swarm algorithm in modeling a flexible beam structure
}

\author{
Rickey Ting Pek Eek ${ }^{1}$, Intan Z. Mat Darus², Shafishuhaza Sahlan ${ }^{3}$, \\ Pakharuddin Mohd Samin ${ }^{4}$, Nik M. R Shaharuddin ${ }^{5}$ \\ ${ }^{1,2}$ Department of Applied Mechanics, Faculty of Mechanical Engineering, 81310, Johor Bahru, Malaysia \\ ${ }^{3}$ Department of Control and Mechatronics Engineering, Faculty of Electrical Engineering, \\ 81310, Johor Bahru, Malaysia \\ ${ }^{4,5}$ Department of Aeronautics, Automotive and Ocean Engineering, Faculty of Mechanical Engineering, \\ Universiti Teknologi Malaysia, 81310, Johor Bahru, Malaysia \\ ${ }^{2}$ Corresponding author \\ E-mail: ${ }^{1}$ rickeyting2012@hotmail.com, ${ }^{2}$ intan@fkm.utm.my, ${ }^{3}$ shafis@fke.utm.my,.pakhar@utm.my, \\ 5ridzuan@mail.fkm.utm.my
}

Received 6 May 2014; received in revised form 11 August 2015; accepted 16 August 2015

DOI https://doi.org/10.21595/jve.2015.15182

\begin{abstract}
The application of System Identification techniques for modeling a flexible beam structure are presented in this paper. The flexible beam has been widely applied in various fields engineering and industrial. However, the flexible structure is easily influenced by unwanted vibration which may lead to fatigue, performance reduction and structure damage. Thus, the unwanted vibration must be controlled and reduced. In order to have a good controller performance for vibration suppression, an appropriate model of flexible beam is required. Hence, to obtain a model of the flexible beam structure, Particle Swarm Optimization (PSO) and Artificial Bee Colony $(\mathrm{ABC})$ are implemented in this study as System Identification techniques. The implementation of PSO and $\mathrm{ABC}$ requires experimental data input and output retrieved from data acquisition from a well-developed experimental test rig via MATLAB Simulink platform. Results obtained are displayed in graphical plots and numerical values. The predicted model is validated via mean square error (MSE) and correlation tests. To represent the dynamic model of the flexible beam structure, model with minimum MSE value and correlation test within $95 \%$ confidence interval is selected as the best fit model. The result shows that PSO algorithm produces better performance compared to ABC algorithm with a 3rd order predicted model that has lowest MSE value and correlation tests within $95 \%$ confidence interval for the beam system.
\end{abstract}

Keywords: flexible beam, particle swarm optimization, system identification, artificial bee colony, MATLAB Simulink.

\section{Introduction}

Process modeling is a technique of achieving mathematical model which describes the behavior of a dynamic system. System Identification is one of the modeling methods used to build the mathematical model using experimental data input and output. System Identification has been developed for many years and different views on the definition of System Identification have been established.

In [1], an equivalent model can be determined from a set of data input and output which is the basic of identification from a group of models for the system under test. In addition, in [2], the identification problem can be summed up as a type of mathematical expression which represents the essential characteristics of an objective system by a model. By understanding the objective system, a useful form of the system can be expressed by using the obtained model. Furthermore, [3] mentioned that three important elements in System Identification are the data, models and standards. It stated that the identification is the process of selecting a model which fits the original data in accordance with a criterion. On top of that, System Identification has been widely applied in linear and nonlinear system $[5,6]$ in different application such as automotive air conditioning [7], flexible plate structure [8], flexible manipulator system [9], and flexible appendage on satellite for autonomous control [10]. 
System Identification has also been implemented with intelligent application technique to search the unknown parameters in modeling. Particle Swarm Optimization (PSO) is an intelligent swarm optimization method regarding the behavior of system in food searching which is proposed by Kennedy and Eberhart in 1995 [22]. PSO technique has been widely applied by researchers to model a system in recent years. The unknown parameters in mathematical model can be obtained effectively and efficiently via PSO technique. In [11], a power system measurement based load is applied with PSO technique to develop a mathematical model of the system. Meanwhile, [12] had implement PSO to IIR adaptive System Identification problem. The PSO technique has been modified and improved which produces a better result compared to the RGA and basic PSO model.

The implementation of PSO in mechanical system for System Identification can be seen in [7], [8] and [9]. Hanim Yatim et al. had model the flexible manipulator system using PSO and RLS [9]. In [9], it showed that PSO has the advantages over RLS in parametric modeling of flexible manipulator system. In addition, Muhammad Sukri Hadi et al. implemented PSO technique to obtain the model of flexible plate structure system with Free-Free-Clamped-Clamped Edge [8]. The PSO model is compared with RLS model and found that PSO model has performed better than RLS model in terms of mean-square-error (MSE), one-step-ahead prediction (OSA) and correlation test within $95 \%$ confidence interval. Furthermore, PSO technique has been applied in modeling of automotive air conditioning system by Md Lazin et al. [7]. An ARX model is developed using PSO and RLS which both models were validated using OSA, MSE and correlation test. The result showed that PSO technique has produce the best ARX model with the lowest MSE value compared to RLS method.

Meanwhile, Artificial Bee Colony (ABC) algorithm is also known as intelligent optimization technique that has been employed in process modeling by several researchers. The concept of $\mathrm{ABC}$ is based on the bees' activities in nectar foraging. The advantages of $\mathrm{ABC}$ algorithm are easy to implement, broad applicability in complex function, robust, able to explore local solutions and global optimizer [16]. ABC algorithm has been studied and enhanced since its proposal introduced by Karaboga in 2005 [17]. The research based on ABC algorithm had been carried out in solving the nonlinear equation [18] and identification of linear system [19] such as linearized power system stabilization [20].

In this paper, a cantilever flexible beam structure is the system that will be manipulated as shown in Fig. 1. The beam structure is one of the types of flexible structures that has been popularly applied in various fields of mechanical engineering, for instance, submarine, airplanes, ships, etc. However, the flexible structure is always influenced by unwanted vibration easily which may lead to drawbacks such as fatigue, instability, structure damage and performance reduction. Therefore, the unwanted vibration must be reduced and controller by an intelligent controller. To achieve the best controller performance, the development of an appropriate and accurate model to represent the beam system is utmost important.

To develop the model of the beam system, Mohammad et al. had successfully implement PSO technique with dynamic spread factor inertia weight in modeling the flexible system [15]. Result showed that PSO technique has the capability to obtain the model of the beam system with a low MSE value and good correlation test. However, the ABC algorithm is still a very new method to model the flexible beam system. Hence, this paper applied PSO and ABC algorithm to obtain the mathematical model of the beam system via System Identification using data acquisition from a well-developed experiment test rig. Both models are compared and validated using MSE, OSA and correlation test within $95 \%$ confidence interval.

In this paper, the introduction and literature review of this work is presented in Section 1. In Section 2, the description of System Identification is discussed in detail. Moreover, the description of PSO and $\mathrm{ABC}$ are both presented in Section 3 and Section 4 respectively. In addition, the description of methodology of the work is discussed in Section 5. Meanwhile, in Section 6, the simulation results and discussion for PSO and ABC are compared and presented. Lastly, the conclusion for this work is presented in Section 7. 


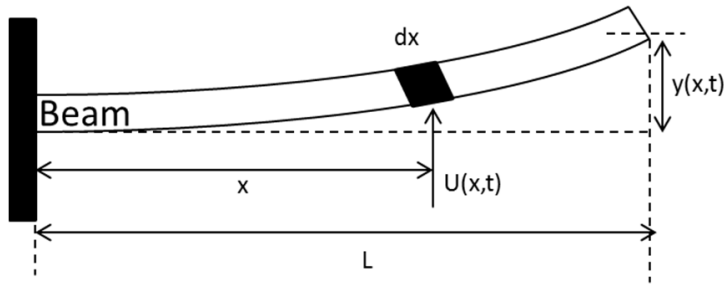

Fig. 1. The cantilever beam system

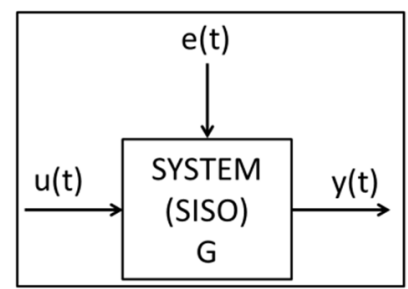

Fig. 2. Concept of a simple black-box model with SISO

\section{System identification}

The modeling of a dynamic system is an important process to describe the characteristic and behavior of a system in term of mathematical equation. To achieve the best controller performance in control system, an appropriate model is required to be developed.

Two types of approaches to obtain the model of a dynamic system are discussed in [4]. It stated that one of the approaches for modeling a system is to create a fundamental model based on knowledge (physics and chemistry) of the system to be modeled. Such model is described as the 'right' model that provides the correct and actual behavior of the system. In addition, it is robust that can be applied repeatedly under any different conditions. However, it is the most difficult and time consumed way in obtaining all of the knowledge regarding the physical and chemistry of the system, especially if the system is complicated.

The second approach for modeling a system is using System Identification by choosing an appropriate mathematical form (or known as transfer function) that represents the behavior of the system via experimental data acquisition. This type of model is known as 'black box' or 'input-output' model which is convenient, simple and easy to manipulate. This model seeks to reproduce the behavior of the system's output if only the set point or input has been changed. The advantage is the model can be developed without prior knowledge about the system; hence, the complicated system can be modeled easily and quickly.

A simple black-box or input-output model is represented in Fig. 2. $u$ and $y$ are both denoted as the input and output of the system respectively. The output system will be changed in response of the change of inputs and time. Assume that $G$ is the plant system, the general transfer function in frequency domain is denoted as $G(s)=Y(s) / U(s)$. In control system, the controller performance depends on the identified plant model, $G(s)$ by using System Identification.

The system identification procedures are as following:

1) Experimental design and setup.

An analytical model of the structure is built and well-developed. All the instrumentation requirements needed are determined to collect the data input and output with prescribed accuracy and spatial resolution.

2) Perform the experiment.

The data input and output are collected from the experiment. The System Identification technique is implemented to the experiment data to obtain the mathematical model of the system.

3) Choosing a suitable model.

The model can be chosen using prior knowledge or trial-and-error. There are various types of model that can be considered as the model in System Identification, for instance, AR, ARX, ARMA, NARX, NARMA, etc.

4) Evaluate and validate the model.

The predicted model can be evaluated and validated by comparing the predicted output with the original output which is called as error. Correlation test can be brought out to test the relationship between the error and input. The best fit model always been selected if only the error is minimum and unbiased correlation test. 


\section{Particle swarm optimization}

In 1995, Kennedy and Eberhart proposed Particle Swarm Optimization (PSO) algorithm which is a stochastic global optimization algorithm regarding the behaviors of the swarms such as flock of birds, schools of fish or swarm of bees in food searching. PSO has been widely used in various fields and application especially in modeling and control.

PSO involves the problem that needs to be solved which the fitness function, $f(x)$ is defined and to be minimized or maximized. A set of particles search their food in a search space repeatedly until the stopping criteria is met. Swarm is the set of potential solutions to the optimization problem where particle is the potential solution in the swarm set. The best solution achieved by the particle and swarm in a neighborhood are called as personal best position (denoted as pbest in Eq. (1)) and global best position (denoted as gbest in Eq. (2)) respectively. The pbest and gbest values found are always being memorized until the end of the searching. The previous memorized solution will be replaced and updated if there is a better solution compared to the previous solution:

pbest $=p_{\text {id }}(t+1)= \begin{cases}p_{i d}(t), & f\left(x_{i d}(t+1)\right) \geq f\left(p_{i d}(t)\right), \\ x_{i d}(t+1), & f\left(x_{i d}(t+1)\right)<f\left(p_{i d}(t),\right.\end{cases}$
gbest $=p_{g d}(t) \in\left\{p_{1 d}, p_{2 d}, \cdots, p_{s d}\right\}=\min \left\{f\left(p_{1 d}(t)\right), f\left(p_{2 d}(t)\right), \cdots, f\left(p_{s d}(t)\right)\right\}$,

where $x_{i d}$ is the current position of the particle.

In PSO algorithm, each particle will be first initialized for its position and velocity randomly in a swarm set. It holds the position as the candidate solution to the problem and the velocity as the flying direction of the particle. The velocity and position will be updated using Eqs. (3) and (4) respectively in each iteration of solution searching process:

$$
\begin{aligned}
& \text { newvelocity }=v_{i d}(t+1) \\
& \quad=v_{i d}(t)+c_{1} r_{1}(t)\left(p_{i d}(t)-x_{i d}(t)\right)+c_{2} r_{2}(t)\left(p_{i d}(t)-x_{i d}(t)\right) \\
& \text { newposition }=x_{i d}(t+1)=x_{i d}+v_{i d}(t+1)
\end{aligned}
$$

where $c_{1}, c_{2}$ are the acceleration constants, $r_{1}$ and $r_{2}$ are the random numbers with the value between 0 and 1. In [11], $c_{1}$ and $c_{2}$ are adjustable experimentally between 1 and 2. For a better control exploitation and exploration, the inertia weight is introduced as in Eq. (5):

$v_{i d}(t+1)=w v_{i d}(t)+c_{1} r_{1}(t)\left(p_{i d}(t)-x_{i d}(t)\right)+c_{2} r_{2}(t)\left(p_{i d}(t)-x_{i d}(t)\right)$.

Inertia weight $w$ serves as the memory of the previous direction to prevent the particle from changing direction drastically [9]. It can be updated using Eq. (6) to avoid $w$ from exceeding the number of iterations:

$w=w_{\max }-\frac{w_{\max }-w_{\min }}{i t e r_{\max }} \times t$

A basic procedure for PSO technique is presented in the flow chart as shown in Fig. 3.

\section{Artificial bee colony}

Artificial Bee Colony (ABC) algorithm involves the behavior of a bee in food foraging. ABC algorithm was proposed by Devis Karaboga in 2005 [17]. It was formed by observing the activities and behaviors of the real bees which were looking for the nectar resources and sharing the amount of nectar resources with the other bees.

In $\mathrm{ABC}$ algorithm, there are three essential components which are food sources, employed 
foragers and unemployed foragers. The food sources represent the solutions to the optimization problems. A forager bee identifies the quality of a food source, such as the taste of its nectar, nearness to the hive, and efficiency of the energy in order to choose a food source [21]. An employed forager acts as an information carrier of a specific food source and shares the information to other bees waiting in the hive. The information contains the distance between the hive and the food source, the direction and the cost of the food source [19]. An unemployed forager who may be an onlooker bee or scout bee looks for a food source to exploit. An onlooker bee attempts to search a food source regarding to the information shared by employed bee. A scout bee searches for a food source randomly without any knowledge and fact.

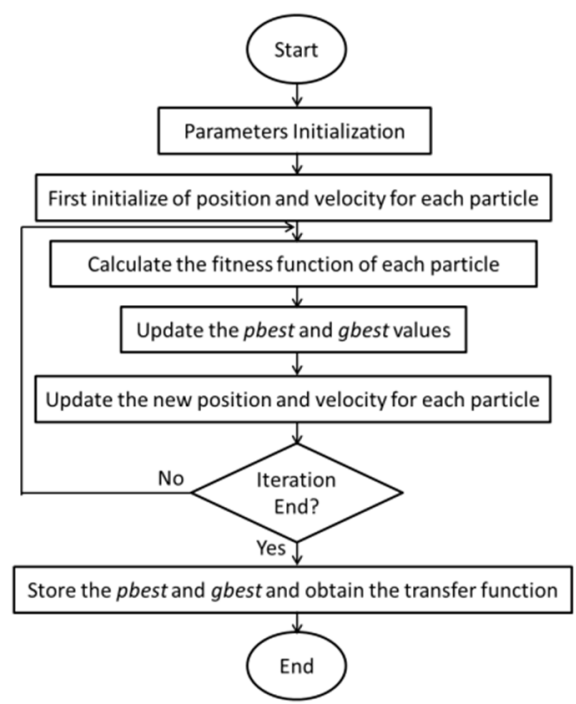

Fig. 3. The flow chart of PSO algorithm

The steps in $\mathrm{ABC}$ algorithm are stated below:

1) Initialization population;

2) Repeat;

3) Place the employed bees on the food source;

4) Evaluate the food source and share to onlooker bees;

5) Place the onlooker bees on the discovered food source based on the information shared by employed bees;

6) Evaluate the food source;

7) Send a scout bee for searching new food source;

8) Memorize the best food source found so far;

9) Until requirements are met.

In the first initialization, a set of food source with a particular range of the variables $x_{i}$ $(i=1,2, \ldots, S N)$ is randomly selected by the bees. The initial amount of nectar source is determined and the best food source is memorized.

In $\mathrm{ABC}$ algorithm, half of the bees in colony are chosen as employed bees and another half for onlooker bees. In the first step of ABC, the employed bees will search a new set of food source using Eq. (7) and evaluate the food via a fitness function in Eq. (8). The information of the food source will be memorized and shared to the onlooker bees waiting in the hive. The onlooker bees observe, evaluate and choose the food source via the dances presented by employed bees:

$v_{i j}=x_{i j}+\phi_{i j}\left(x_{i j}-x_{k j}\right)$,

where $k \in(i=1,2, \ldots, S N), j \in\{1,2, \ldots, D\} . k$ is generated by $\operatorname{int}(\operatorname{rand}, S N)+1$ while $S N$ is 
the number of food source. $j$ is one-dimensional variable in $D$ dimensional vector. Both $k$ and $j$ are randomly generated with $k \neq i . \phi_{i j}$ is a random number between -1 and 1 .

The fitness function for the food evaluation after the discovery of the source is denoted as in Eq. (8):

FitnessFunction $= \begin{cases}\frac{1}{f_{i t_{i}+1}}, & f_{i}>0, \\ 1+f i t_{i}, & f_{i} \leq 0,\end{cases}$

where $f_{i}$ is the cost function of solution $i$.

In the second step of $\mathrm{ABC}$, onlooker bees make a resource choice depending on the probability value in Eq. (9) associated with the food source:

Probability, $P=\frac{\text { fit }_{i}}{\sum_{n=1}^{S N} f i t_{i}}$,

where $f i t_{i}$ - fitness value of solution $i$.

The onlooker bees will search for new solutions within the resource chosen using Eq. (7) and evaluate the food via fitness function in Eq. (8). The greedy selection process is applied if there is a better food source compared to the previous one. The previous food source will be replaced by the new better food source.

In the third step, a scout bee has been selected from the abandoned employed bee. If the abandoned source is unable to be improved anymore, the abandoned employed bee will become as a scout bee to identify a new resource in the search space without any prior knowledge and fact.

Assume that the abandoned source is $x_{i}$ with $j \in\{1,2, \ldots, D\}$, then the scout discovers a new food source to replace the previous $x_{i}$ based on Eq. (10):

$x_{i}^{j}=x_{\min }^{j}+\operatorname{rand}[0,1]\left(x_{\max }^{j}-x_{\min }^{j}\right)$.

\section{Methodology}

\subsection{Experiment setup}

In this paper, a cantilever beam is selected as flexible beam structure as shown in Fig. 4 . The cantilever beam is made of aluminum with the length $600 \mathrm{~mm}$, thickness $1 \mathrm{~mm}$ and width $53 \mathrm{~mm}$. The parameters regarding the physical measurement of cantilever beam are listed in Table 1 . The beam has been equally divided into 20 segments as shown in Fig. 5. The divided segment is used to determine the actuators' and sensors' location. In this paper, disturbance actuator, control actuator, detection sensor and observation sensor are placed at the segments between 8th and 9th, $10 \mathrm{~h}$ and 11 th, 9th and 10th, 19th and 20th respectively as shown in the schematic diagram Fig. 6. The base is built to ensure the beam structure is clamped strongly and stable in order to prevent any immeasurable noise to disturb the beam system.

The beam system applied in this paper is presented in the schematic diagram Fig. 6. This system requires several important components which are piezoelectric patches, amplifiers, DAQ connector block, DAQ card, personal computer (PC) and software platform using MATLAB SIMULINK. These required components with each specification are listed in Table 2 . In this system, four piezoelectric patches with the same types of PI P-876.A11 are required as the sensors and actuators which are mounted on both sides of the beam structure. The disturbance actuator is used to vibrate the beam while the control actuator is used to control and reduce the vibration of the beam based on the controller output given. In addition, the detection sensor is used to detect the input that beam experienced while the observation sensor is used to observe the motion and vibration of the beam structure at a certain point of the beam structure. However, in this paper, the 
control actuator is mounted at the beam for modeling purpose only.

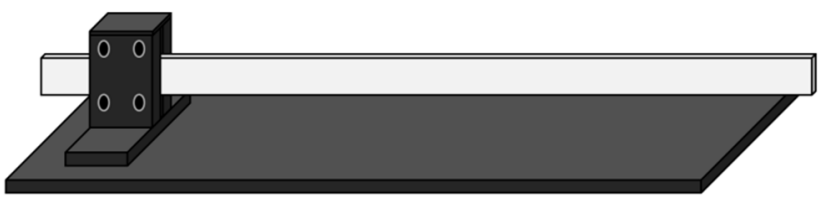

Fig. 4. The cantilever beam system

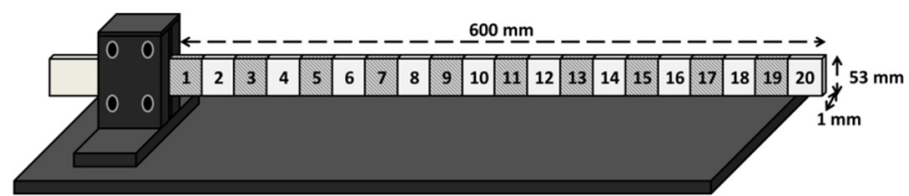

Fig. 5. Physical measurement of cantilever beam with 20 segments division

Apart from that, another important component in this system is amplifier. The signals generated from PC and piezoelectric sensors are amplified by amplifiers in voltage. Furthermore, a DAQ connector block is used as a signal conditioning block which consists of ADC and DAC. The block is connected to the DAQ card and amplifiers. The DAQ card is installed in the PC with port PCI. On top of that, another important component used in this system is the software platform. In this paper, the MATLAB SIMULINK blocks are developed to collect the data input and output from experiment for modeling purpose.

There are four piezoelectric patches mounted at the sides of beam structure as shown in the schematic diagram Fig. 6 . Each piezoelectric patch requires an amplifier for signal amplification. This system starts with the generation of disturbance signal from the PC. The signal is sent to actuator after digital-analog conversion in DAQ system. In this paper, a sinusoidal signal is selected as the disturbance to the beam system. The signal is transmitted in voltage from DAQ connector block to the amplifier E-835 and then to the piezoelectric actuator. The piezoelectric patch will start bending after receiving the voltage signal at the disturbance point on the beam and thus to vibrate the beam. Meanwhile, the piezoelectric sensors will sense the vibration at the detection and observation points. The signals sensed are transmitted to the DAQ system in order to allow the computer understands and read the signal retrieved after the amplification using amplifiers E-413. The signals can be displayed in MATLAB SIMULINK scope to allow the user observes the vibration and stores the data input and output for System Identification purpose.

Table 1. Specification of cantilever beam structure

\begin{tabular}{|l|c|}
\hline Beam structure & Specification \\
\hline Material & Aluminum \\
\hline Length & $600 \mathrm{~mm}$ \\
\hline Width & $53 \mathrm{~mm}$ \\
\hline Thickness & $1 \mathrm{~mm}$ \\
\hline
\end{tabular}

Table 2. Tools requirement with specification and functionality

\begin{tabular}{|l|c|c|c|}
\hline \multicolumn{1}{|c|}{ Tools } & Quantity & Specification & Functionality \\
\hline Piezoelectric patches & 4 & PI P-876.A11 & Sensor and actuator \\
\hline Amplifiers (1) & 2 & E-835 DuraAct & Amplification \\
\hline Amplifiers (2) & 2 & E-413 DuraAct & Amplification \\
\hline DAQ Card & 1 & NI-DAQ PCI-6259 & Data acquisition \\
\hline DAQ connector block & 1 & SCC-68 M Series & Signal conditioning \\
\hline Personal computer & 1 & HP Intel I7-3770 & Computation \\
\hline Beam & 1 & Aluminum & Flexible structure \\
\hline Software & 1 & MATLAB R2012A & Graphical user interface \\
\hline
\end{tabular}


In this paper, data acquisition is simulated using MATLAB SIMULINK Toolbox which is installed in the PC and integrated to the experimental test rig. The data acquisition system for obtaining the data input and output is shown in Fig. 7. The main components of data acquisition system are analog input, analog output, low pass filter and disturbance source. The analog input is connected to the sensors where the signals of vibration are retrieved. Apart from that, the analog output is connected to disturbance and control actuators that used to vibrate and control the beam structure respectively.

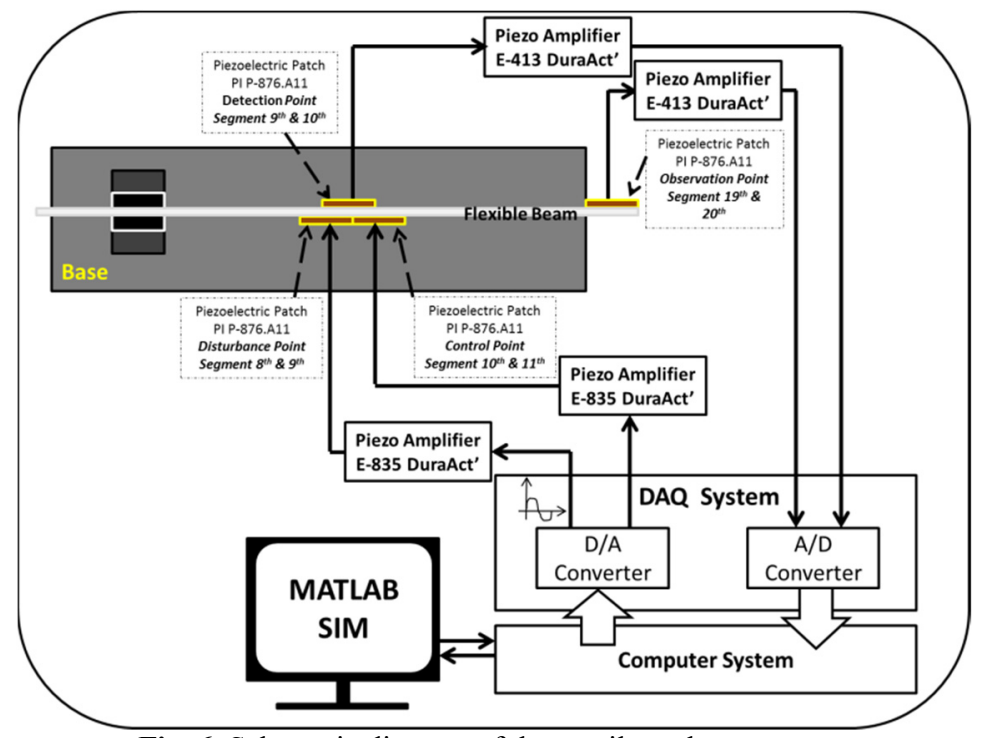

Fig. 6. Schematic diagram of the cantilever beam system
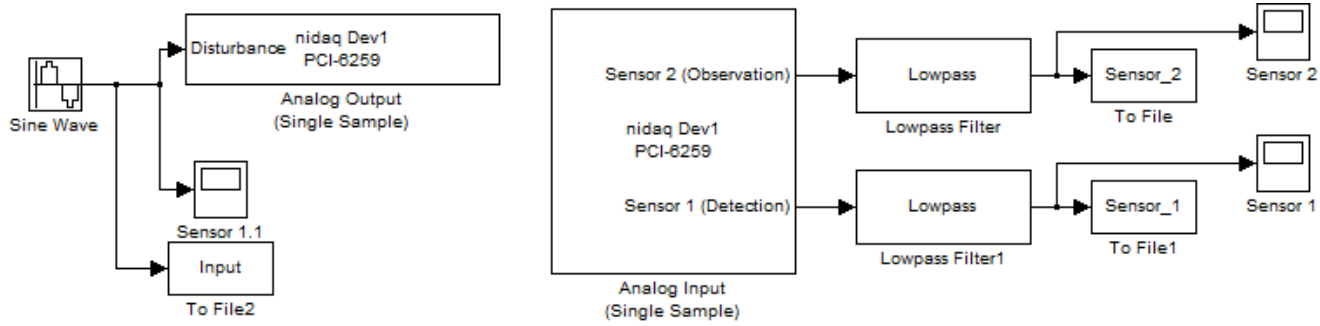

Fig. 7. The block diagram of data acquisition system using Matlab Simulink toolbox

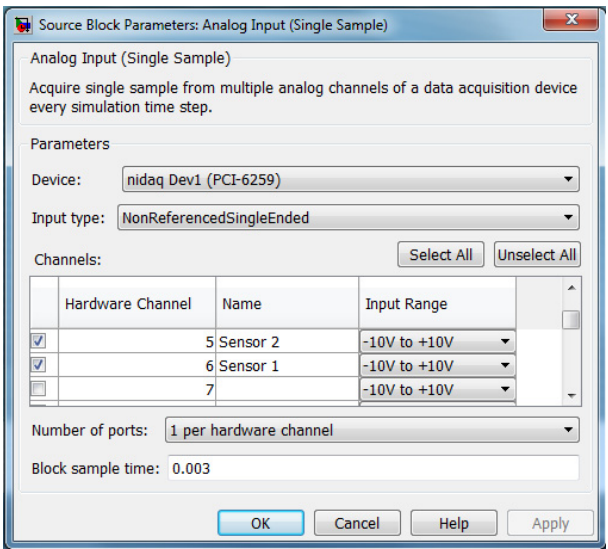

Fig. 8. The settings of analog input

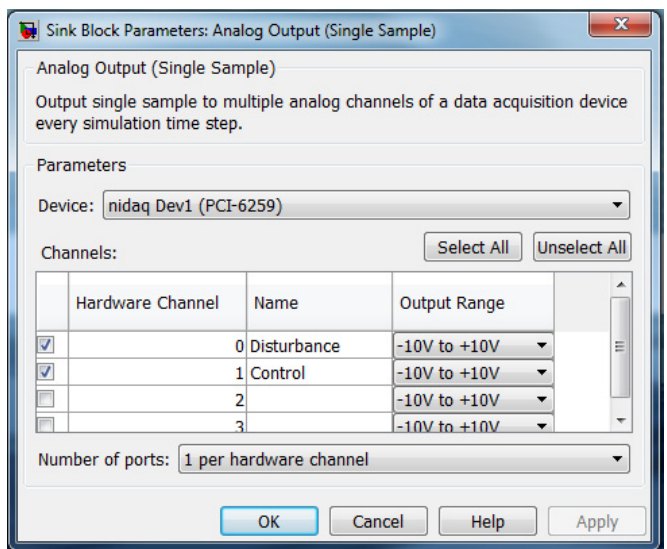

Fig. 9. The settings of analog output 
The PCI-6259 National Instrument DAQ card is used for data acquisition system. The settings of the analog input and output are shown in Fig. 8 and Fig. 9 respectively. The device for analog input and output is nidaq Dev1 (PCI-6259) with the hardware channel AI6 and AI5, and AO0 respectively. The input type for analog input is set as "Non Referenced Single Ended" (NRSE) with the input range of $-10 \mathrm{~V}$ to $+10 \mathrm{~V}$ and 0.003 sampling time. The low pass filter is used to eliminate the noise caused by the wiring connection and electrical components in the system.

In this paper, a $4 \mathrm{~V}$ sine wave with frequency $2 \mathrm{~Hz}$ is generated as the force disturbance to the beam system as shown in Fig. 10. The disturbance signal is sent via the analog output to the actuator.

The motion of the beam that sensed from piezoelectric sensors can be observed from the scopes of sensor 1 and sensor 2 in the block diagram Fig. 7. The data input and output are saved in mat file for System Identification purpose.

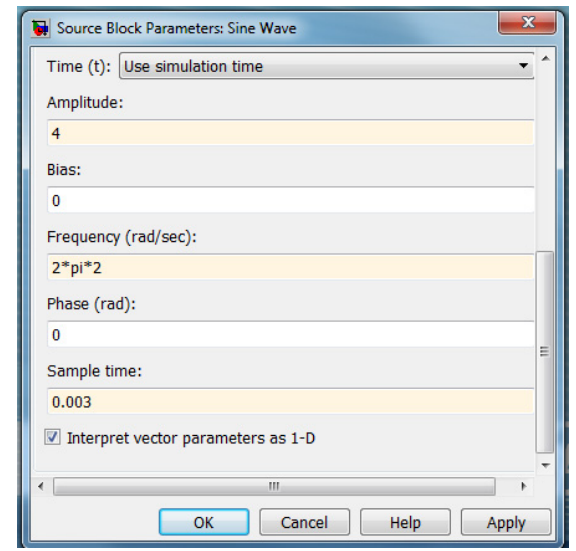

Fig. 10. The settings of sine wave disturbance

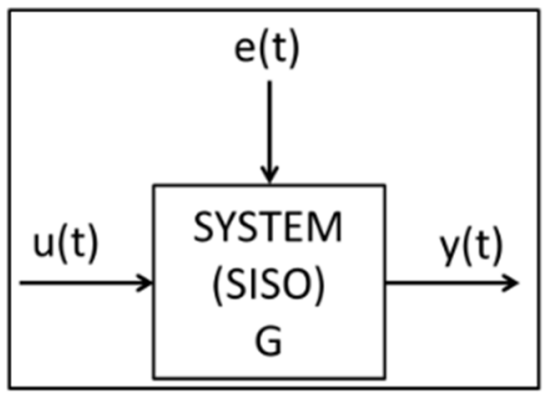

Fig. 11. The SISO system $\mathrm{G}$

\subsection{System identification}

In System Identification, the estimation of unknown parameters is important based on the experiment data input and output in order to develop an accurate model of the system with appropriate order number and parameters. The autoregressive with exogenous input (ARX) model is applied in this paper.

The ARX model is denoted as in Eq. (11):

$y(t)=\frac{B\left(z^{-1}\right)}{A\left(z^{-1}\right)} u(t)+\frac{e(t)}{A\left(z^{-1}\right)}$

The ARX model $G$ in zero order hold is denoted as in Eq. (12):

$G=\frac{y(t)}{u(t)}=\frac{B\left(z^{-1}\right)}{A\left(z^{-1}\right)}$

Assume that the white noise, $e(t)$ is zero:

$A\left(z^{-1}\right)=1+a_{1} z^{-1}+\cdots+a_{n a} z^{-n a}$,

$B\left(z^{-1}\right)=b_{1} z^{-1}+\cdots+b_{n b} z^{-n b}$,

where $z^{-1}$ is the backshift operator while the number of coefficients of denominator $n_{a}$ and numerator $n_{b}$ are equal in this paper.

A Single-Input-Single-Output (SISO) system is applied as shown in Fig. 11. In this system, a 
signal input $u(t)$ is applied with $t \in[1,2, \ldots, N]$. The output measured, $y(t)$ is obtained experimentally with $t \in[1,2, \ldots, N]$.

Assume that white noise, $e(t)$ is zero, the estimated output, $\hat{y}(t)$ can be obtained using Eq. (15):

$\hat{y}(t)=\phi(t) \theta$

where:

$\phi(t)=[-y(t-1),-y(t-2), \cdots, y(t-n a) \cdots-u(t-1),-u(t-2), \cdots,-u(t-n b)]$.

In this paper, $n a$ and $n b$ are equal to the order number. $\theta$ is the estimation parameters vector with $\theta=\left[a_{1}, a_{2}, \cdots, a_{n a}, b_{1}, b_{2}, \cdots b_{n b}\right]$ that can be obtained using optimization technique to develop the estimated ARX model G of the beam system in Eq. (12).

After estimating the unknown parameters for ARX model G, the estimated ARX model is validated by using error prediction, $e$ and mean-square-error (MSE) methods. The error prediction between the output of the estimated model and experimental data, and MSE value can be calculated as denoted in Eq. (17) and Eq. (18) respectively:

$e=\hat{Y}_{\text {estimated }}-Y_{\text {Experimental }}$,

$M S E=\frac{1}{n} \sum_{i=1}^{n}\left(\hat{Y}_{i}-Y_{i}\right)^{2}$.

MSE is a network performance function that measures the network's performance according to the mean of squared errors. In Eq. (18), $\hat{Y}$ is a vector of $\mathrm{n}$ predictions and $Y$ is the vector of the true values. In this paper, the MSE value is obtained by using MATLAB code ' $m s e(\cdot)$ '.

In this paper, Particle Swarm Optimization and Artificial Bee Colony algorithms will be implemented to obtain the estimation parameters $\theta$ in order to develop an estimated ARX model. The estimated model with minimum values of error prediction and MSE will be chosen as the best fit model for control purpose.

\subsection{Particle swarm optimization}

Particle Swarm Optimization algorithm is simulated using MATLAB environment in this paper. Next, the numbers of swarm sets are determined based on the number of estimated parameters, $N_{s}=$ OrderNumber $\times 2$.

Each swarm set represents one estimated parameter for numerator or denominator. The numbers of swarm sets of estimated parameters for numerator and denominator are the same: $\left(n a=n b=N_{s} / 2\right)$.

For instance, assume that the algorithm consists of 10 swarm sets; 5 sets of the swarm are used to estimate the denominator parameters and another 5 sets for numerator parameters. Each particle holds the information of position in the search space. The number of particle for numerator $N_{P n}$ and denominator $N_{P d}$ are determined by 2 dimensional $i \times j$ of a swarm set as in Fig. 12 .

Estimated parameters vector:

$$
\theta=\left[\begin{array}{l}
a_{1} \\
\vdots \\
a_{n a} \\
b_{1} \\
\vdots \\
b_{n b}
\end{array}\right] \rightarrow\left[\begin{array}{l}
j_{1} \\
\vdots \\
j_{N_{P d}} \\
i_{1} \\
\vdots \\
i_{N_{P n}}
\end{array}\right] .
$$


In the first initialization, each particle is given a random position around the origin $(0,0)$ as in Fig. 13 and solves the fitness function for the first time to obtain the first best particle ("pbest") and global ("gbest") position. After that, each particle in every swarm set will find a new position in the following iteration based on the new velocity obtained from Eq. (3). The new position for each particle will be used to solve the fitness function again. The values of "pbest" and "gbest" will be updated based on the best value obtained from fitness function. In the end of iteration, all the particles in each swarm sets will gather at the same position which is the best optimal solution to the fitness function.

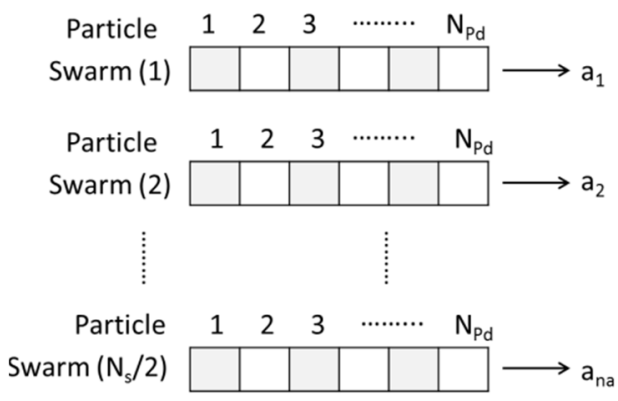

a) Denominator

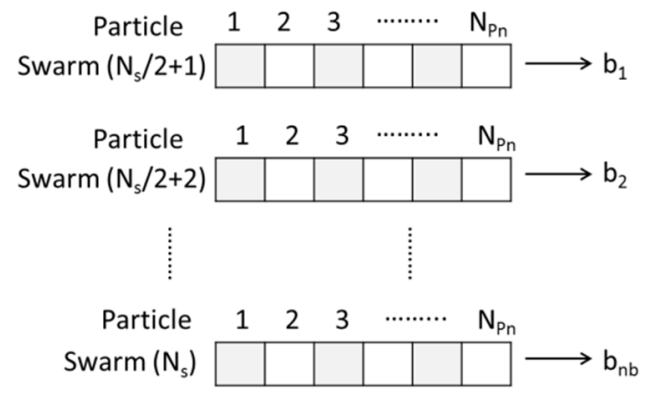

b) Numerator

Fig. 12. The structure of the swarms in PSO

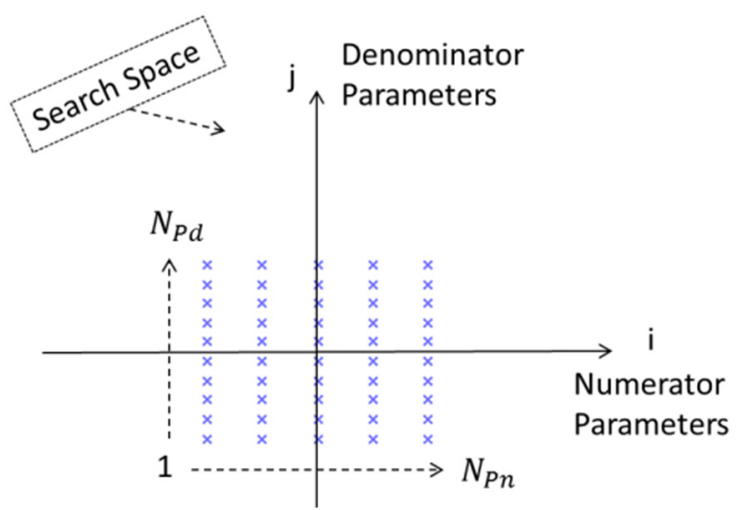

Fig. 13. First initialization of swarms' position

\subsection{Artificial bee colony}

Artificial Bee Colony algorithm is simulated in MATLAB environment to obtain the mathematical model of a flexible beam structure. The experimental data input and output are collected and imported into MATLAB. The parameters required to be determined in $\mathrm{ABC}$ algorithm are listed in Table 3.

Table 3. The parameters required in $\mathrm{ABC}$ algorithm

\begin{tabular}{|c|c|c|c|}
\hline No. & Parameters & No. & Parameters \\
\hline 1. & Size of colony & 6. & Range of search space, $L$ \\
\hline 2. & Number of food source & 7. & Limit trial number \\
\hline 3. & Maximum cycle & 8. & Number of employed bees \\
\hline 4. & Sampling time & 9. & Number of onlooker bees \\
\hline 5. & Model order number & & \\
\hline
\end{tabular}

The algorithm starts with the initialization of the food source. The food source is set initially random in the particular range of search space. The initial food source will be evaluated and 
determined via the cost function and fitness function. The mean-square-error (MSE) of the error between predicted data and experimental data will be the cost function for ABC algorithm in this paper. The best food source found will be stored in the memory.

$\mathrm{ABC}$ algorithm consists of two phases which are employed bee phase and onlooker bee phase. In the employed bee phase, each employed bee holds the solutions for the values of numerator $\left(a_{1}\right.$, $\left.a_{2}, \ldots, a_{n a}\right)$ and denominator $\left(b_{1}, b_{2}, \ldots, b_{n b}\right)$ as indicated in Fig. 14(a).

Each employed bee will update its food source randomly using Eq. (7) and evaluate it via fitness function in Eq. (8). If the random food source from Eq. (7) is exceeding the particular range of search space, it will be randomly picked repeatedly until it is obtained in the desired range. If there is a better fitness value obtained compared to the previous, that particular food source will be updated and stored. The information of food source will be shared with the onlooker bees.

In onlooker bee phase, the onlooker bees will select the food source regarding to the probability in Eq. (9). Each onlooker bee has the solutions for the values of numerator $\left(a_{1}, a_{2}, \ldots, a_{n a}\right)$ and denominator $\left(b_{1}, b_{2}, \ldots, b_{n b}\right)$ as in Fig. 14(b).

Each onlooker bee will update its food source randomly using Eq. (7) and evaluate it via fitness function in Eq. (8). If the random food source from Eq. (7) is exceeding the particular range of search space, it will be randomly picked repeatedly until it is obtained in the desired range. If there is a better fitness value obtained compared to the previous, that particular food source will be updated and stored. In this paper, if the food source consists of a better solution compared to the previous, it will be stored in the memory known as "GlobalParams".

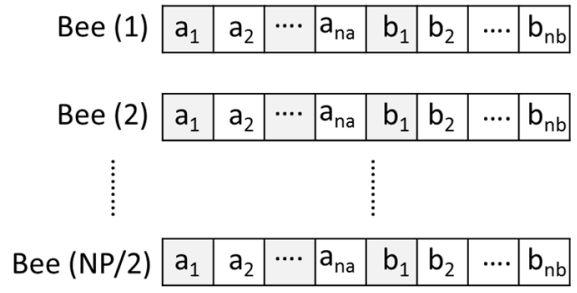

a) Employed bee
Bee (1) \begin{tabular}{|l|l|l|l|l|l|l|l|}
$\mathrm{a}_{1}$ & $\mathrm{a}_{2}$ & $\cdots$ & $\mathrm{a}_{\text {na }}$ & $\mathrm{b}_{1}$ & $\mathrm{~b}_{2}$ & $\cdots$ & $\mathrm{b}_{\mathrm{nb}}$ \\
\hline
\end{tabular}

Bee (2) \begin{tabular}{|l|l|l|l|l|l|l|l|}
\hline$a_{1}$ & $a_{2}$ & $\cdots$ & $a_{n a}$ & $b_{1}$ & $b_{2}$ & $\cdots$ & $b_{n b}$ \\
\hline
\end{tabular}

Bee (NP/2) \begin{tabular}{|l|l|l|l|l|l|l|l|}
$\mathrm{a}_{1}$ & $\mathrm{a}_{2}$ & $\cdots$ & $\mathrm{a}_{\mathrm{na}}$ & $\mathrm{b}_{1}$ & $\mathrm{~b}_{2}$ & $\cdots$ & $\mathrm{b}_{\mathrm{nb}}$ \\
\hline
\end{tabular}

b) Onlooker bees

Fig. 14. The structure of employed bee and onlooker bees

However, if there is a food source that has been abandoned exceeds the limit trial numbers, the employed bee which holds that abandoned food source will become a scout bee. It will search a new random food source using Eq. (10) without any knowledge and fact. The fitness of food found will be evaluated using Eq. (8). If there is a better fitness value compared with the previous one, the abandoned food source will be stored in the memory.

\section{Results and analysis}

\subsection{Experimental data}

In the first step of this work, a $4 \mathrm{~V}$ sinusoidal input with the frequency $2 \mathrm{~Hz}$ as shown in Fig. 15 is transmitted to the system as the disturbance force to vibrate the beam.

This system has been simulated for 5920 iterations data before storing the detection and observation signals. The detection signal that sensed by sensor 1 as shown in Fig. 16(a) is the force that beam experienced which will be the input for the beam system. The vibration of the beam at segment 19th and 20th is plotted in the Fig. 16(b) as observation signal and output of the beam system via sensor 2 .

\subsection{Particle swarm optimization}

In PSO technique, all the parameters required are set as in the Table 4. The PSO algorithm is 
simulated using MATLAB coding which produces the results in graphical plots and numerical values. The system is simulated from 2 nd to 10 th order number and comparison of validation result for each order number is showed in the Table 5.

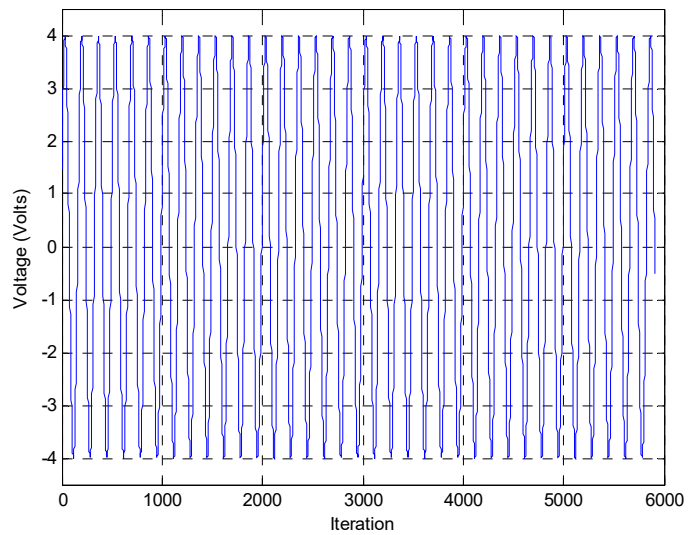

Fig. 15. The disturbance input with sinusoidal signal

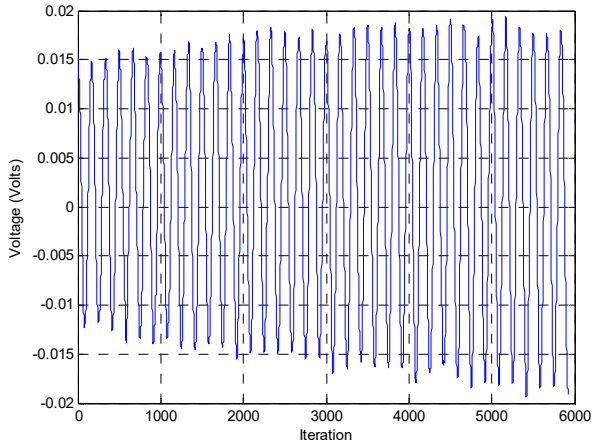

a) Detection of the input disturbance

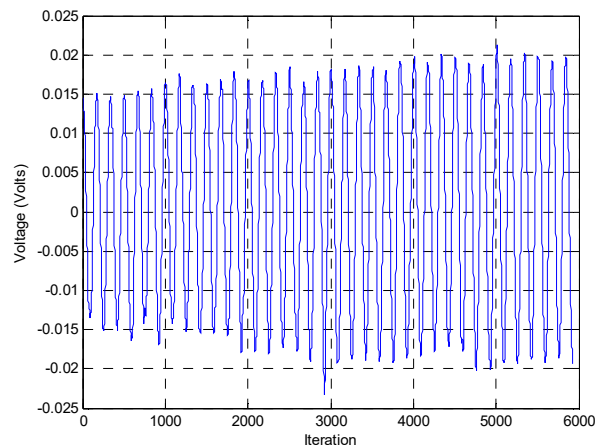

b) Obdervation of the output vibration

Fig. 16. a) The disturbance sensed at detection point on the beam, b) the vibration of the beam at 19 th and 20th segments

From the results obtained in Table 5, it can be observed that an 8th order model yields good result in term of correlation tests which are within $95 \%$ confidence interval. However, a 3rd order model is considered as the best fit model of the flexible beam system in this work. This is because it yields a stable model with the lowest MSE value with $1.9016 \times 10^{-10}$ although its correlation tests are slightly exceeding $95 \%$ interval confidence.

In addition, the comparison of estimated data and original data of beam system in time domain is plotted in Fig. 17. The result shows that a 3rd order system can yield a predicted output that almost similar to the real output of the beam system.

The vibration modes for predicted model and original data output are compared and plotted in Fig. 18. Result shows that the 3 rd order predicted model has the same natural frequencies with the original system in the first 2 vibration modes.

Table 4. The parameters required in PSO algorithm

\begin{tabular}{|l|c|}
\hline \multicolumn{1}{|c|}{ Parameters } & Values \\
\hline Sampling time, $d t$ & 0.003 \\
\hline Correction factor, $b_{1}=b_{2}$ & 2.0 \\
\hline Number of iteration & 1500 \\
\hline Size particle & $20 \times 30$ \\
\hline Range of inertia & $0.15-0.95$ \\
\hline
\end{tabular}


2245. IMPLEMENTATION OF SWARM ALGORITHM IN MODELING A FLEXIBLE BEAM STRUCTURE.

Rickey Ting Pek Eek, Intan Z. Mat Darus, Shafishuhaza Sahlan, Pakharuddin Mohd Samin, Nik M. R Shaharuddin

Table 5. The comparison of MSE values and correlation test with different order number for PSO model

\begin{tabular}{|c|c|c|c|}
\hline Order & MSE & ACR & CCR \\
\hline 2 & $1.3970 \times 10^{-9}$ & 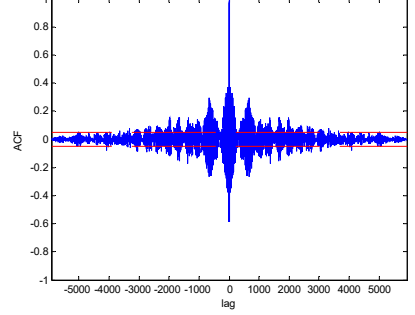 & 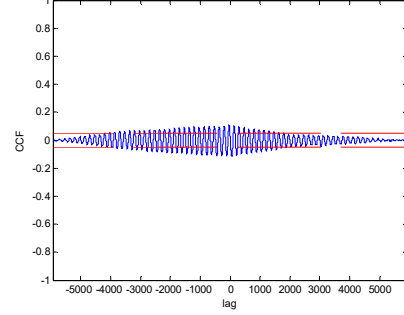 \\
\hline 3 & $1.9016 \times 10^{-10}$ & 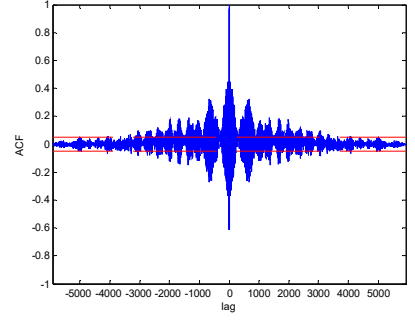 & \begin{tabular}{c|c|c|}
0.8 \\
0.8 \\
0.4 \\
0.4 \\
0.2
\end{tabular} \\
\hline 4 & $1.6879 \times 10^{-9}$ & 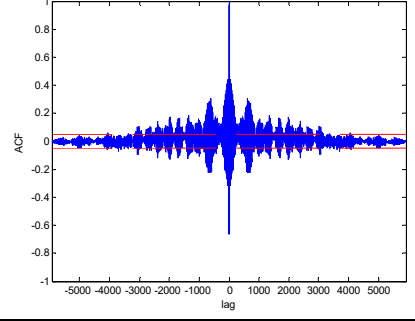 & 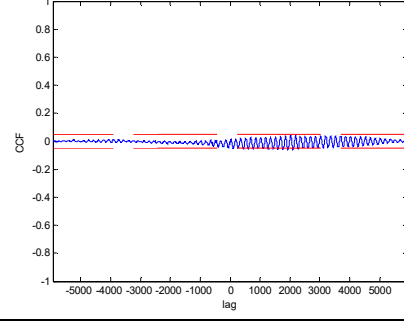 \\
\hline 5 & $4.5122 \times 10^{-9}$ & 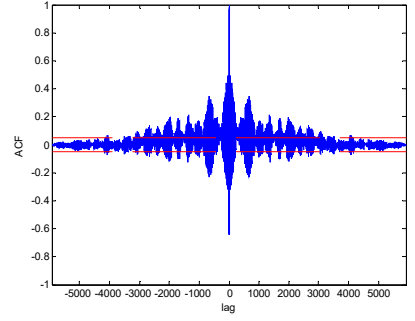 & 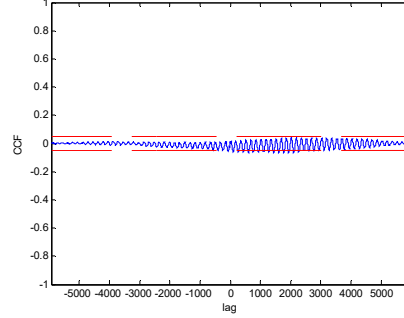 \\
\hline 6 & $1.4880 \times 10^{-9}$ & 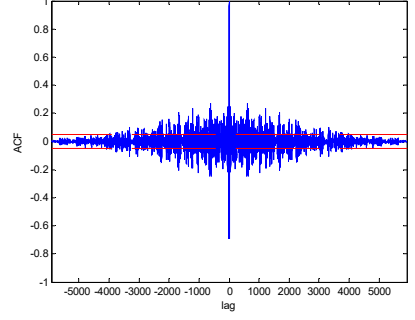 & 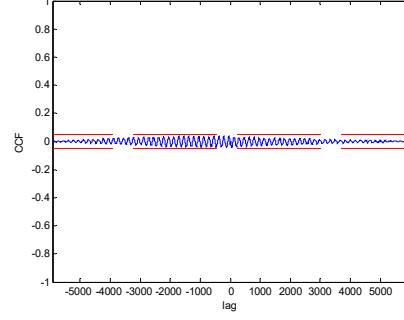 \\
\hline 7 & $2.2448 \times 10^{-9}$ & 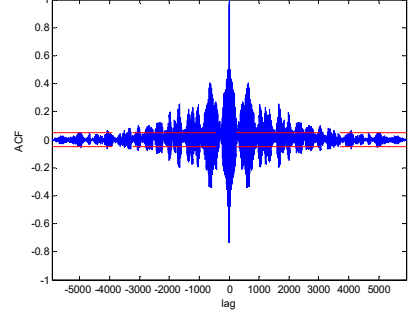 & 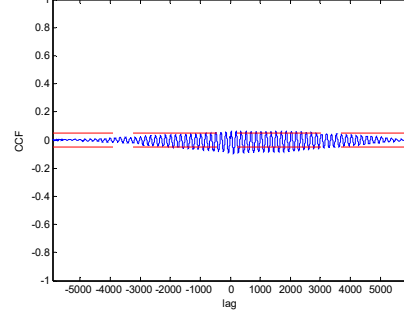 \\
\hline
\end{tabular}




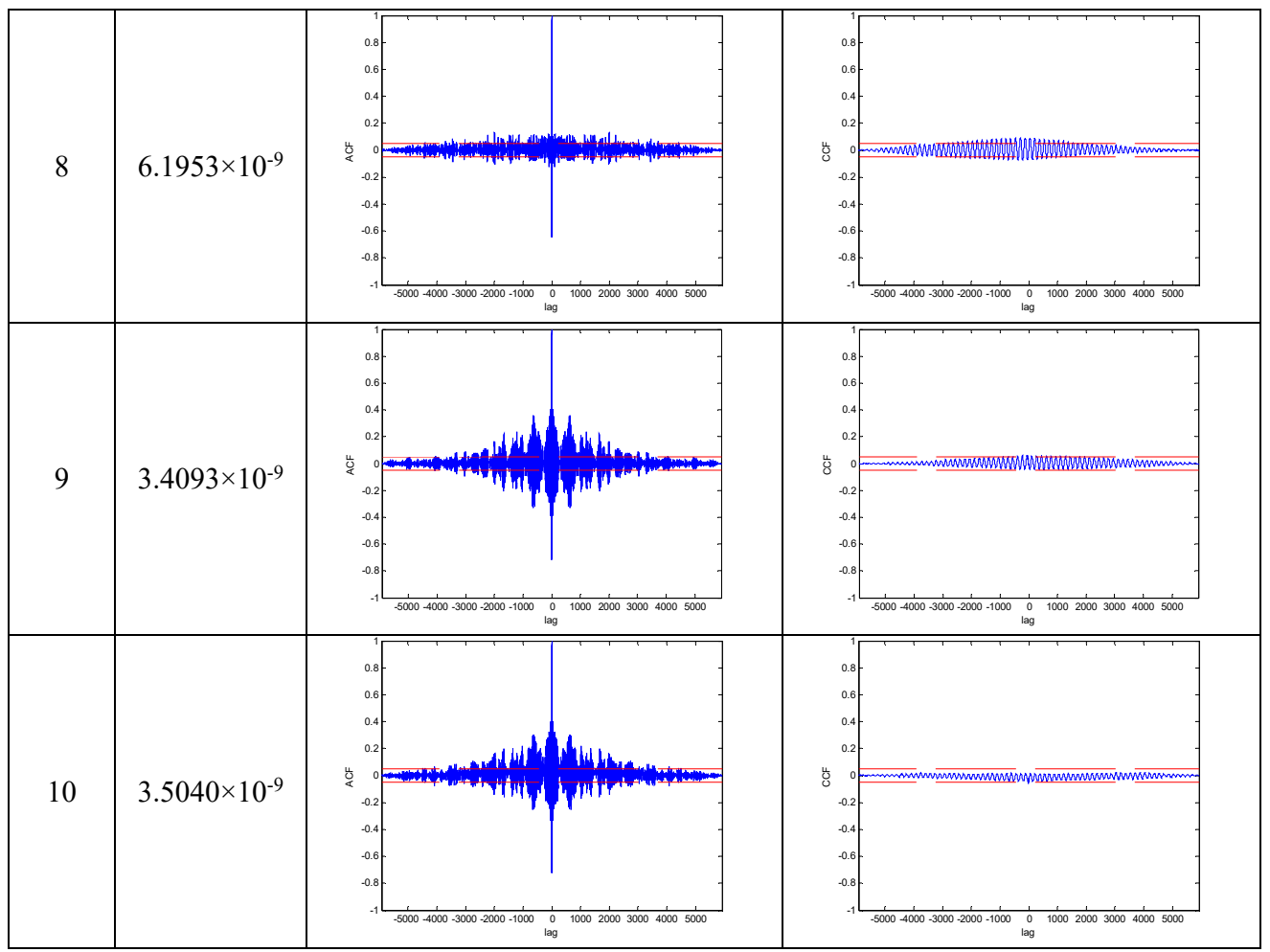

The ARX model $G_{P S O}$ with 3rd order system in zero order hold is presented in Eq. (20). The 3rd order model can be selected for controller design with the PSO application as the model is stable and closed to the real beam system:

$G_{P S O}=\frac{0.2489 z^{-1}-0.4296 z^{-2}+0.1835 z^{-3}}{1-2.415 z^{-1}+1.907 z^{-2}-0.4901 z^{-3}}$.

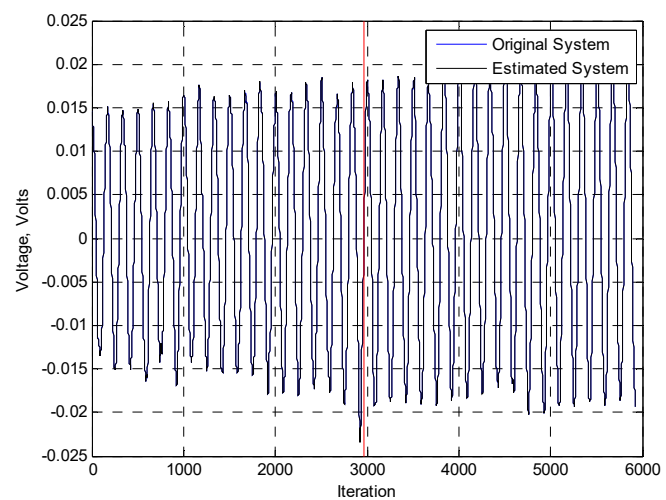

Fig. 17. The vibration of the beam in time domain modeled by PSO algorithm

\subsection{Artificial bee colony}

In $\mathrm{ABC}$ technique, all the parameters required are set as in the Table 6. The ABC algorithm is simulated using MATLAB coding which produces the results in graphical plots and numerical values. The system is simulated from 2 nd to 10 th order number and comparison of validation 
result for each order number is showed in the Table 7.

From the results obtained in Table 7, it can be observed that a 10th order model yields good result in term of correlation tests which are within $95 \%$ confidence interval. However, a 2nd order model is considered as the best fit model of the flexible beam system in this work as it yields a stable model with the lowest MSE value with $2.1723 \times 10^{-9}$ although its correlation tests are exceeding $95 \%$ interval confidence.

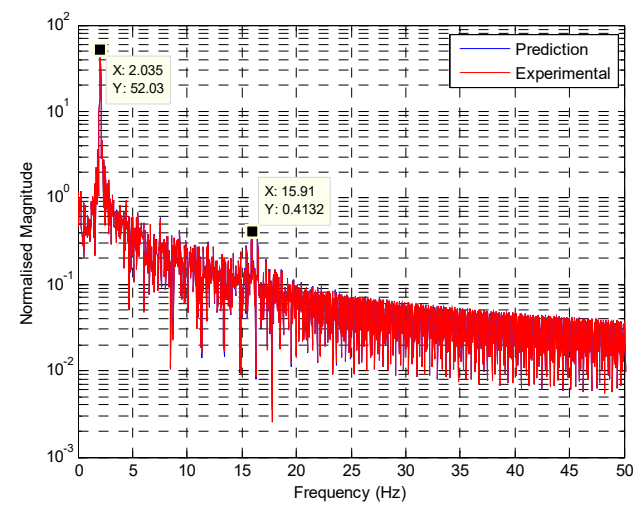

Fig. 18. The comparison of vibration modes between the predicted model and the real system

Table 6. The parameters required in $\mathrm{ABC}$ algorithm

\begin{tabular}{|l|c|}
\hline \multicolumn{1}{|c|}{ Parameters } & Values \\
\hline Sampling time, $d t$ & 0.003 \\
\hline Number of population & 300 \\
\hline Maximum cycle & 1500 \\
\hline Number of employed bees & 150 \\
\hline Number of onlooker bees & 150 \\
\hline Limit of trial number & 15 \\
\hline Range for searching area & {$[$ rand $* 2.5]+0.5$} \\
\hline
\end{tabular}

In addition, the comparison of estimated data and original data of beam system in time domain is plotted in Fig. 19. The result shows that a 2 nd order system can yield a predicted output that almost similar to the real output of the beam system.

The vibration modes for predicted model and original data output are compared and plotted in Fig. 20. Result shows that the 2 nd order predicted model has the same natural frequencies with the original system in the first 2 vibration modes.

The ARX model $G_{A B C}$ with 2nd order system in zero order hold is presented in Eq. (21):

$G_{A B C}=\frac{0.347 z^{-1}-0.3432 z^{-2}}{1-1.673 z^{-1}+0.6763 z^{-2}}$.

From the results obtained using PSO and ABC, it can be seen that both of the models have the same natural frequencies for the first 2 vibration modes. In addition, both models consist of a very low MSE value with low order number. Furthermore, both of the 3rd order PSO model and 2nd order ABC model produce stable system. However, in terms of MSE value and correlation tests, the 3rd order PSO model yields the lowest MSE value of $1.9016 \times 10^{-10}$ and correlation tests within $95 \%$ confidence interval. Meanwhile, the correlation tests of 2 nd order ABC model show that the behavior of the model has less significant correlation between the input and residual compared to 3rd order PSO model. Hence, the 3rd order PSO model is considered as the most suitable model to represent the beam system in this paper. 
2245. IMPLEMENTATION OF SWARM ALGORITHM IN MODELING A FLEXIBLE BEAM STRUCTURE.

Rickey Ting Pek EeK, Intan Z. Mat Darus, ShafishuhaZa Sahlan, Pakharuddin Mohd Samin, NiK M. R Shaharuddin

Table 7. The comparison of MSE values and correlation test with different order number for ABC model

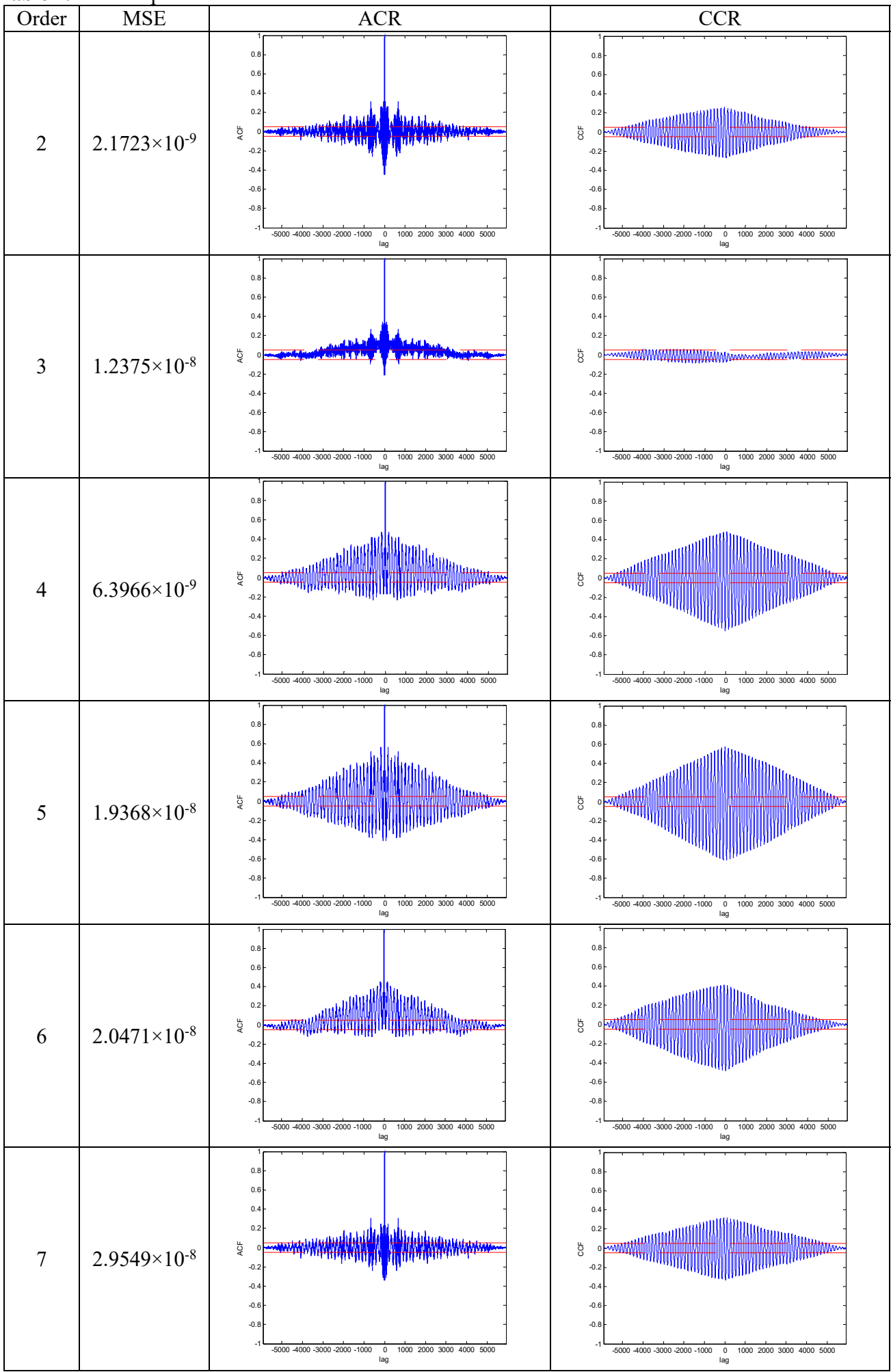


2245. IMPLEMENTATION OF SWARM ALGORITHM IN MODELING A FLEXIBLE BEAM STRUCTURE.

Rickey Ting Pek Eek, Intan Z. Mat Darus, Shafishuhaza Sahlan, Pakharuddin Mohd Samin, Nik M. R Shaharuddin
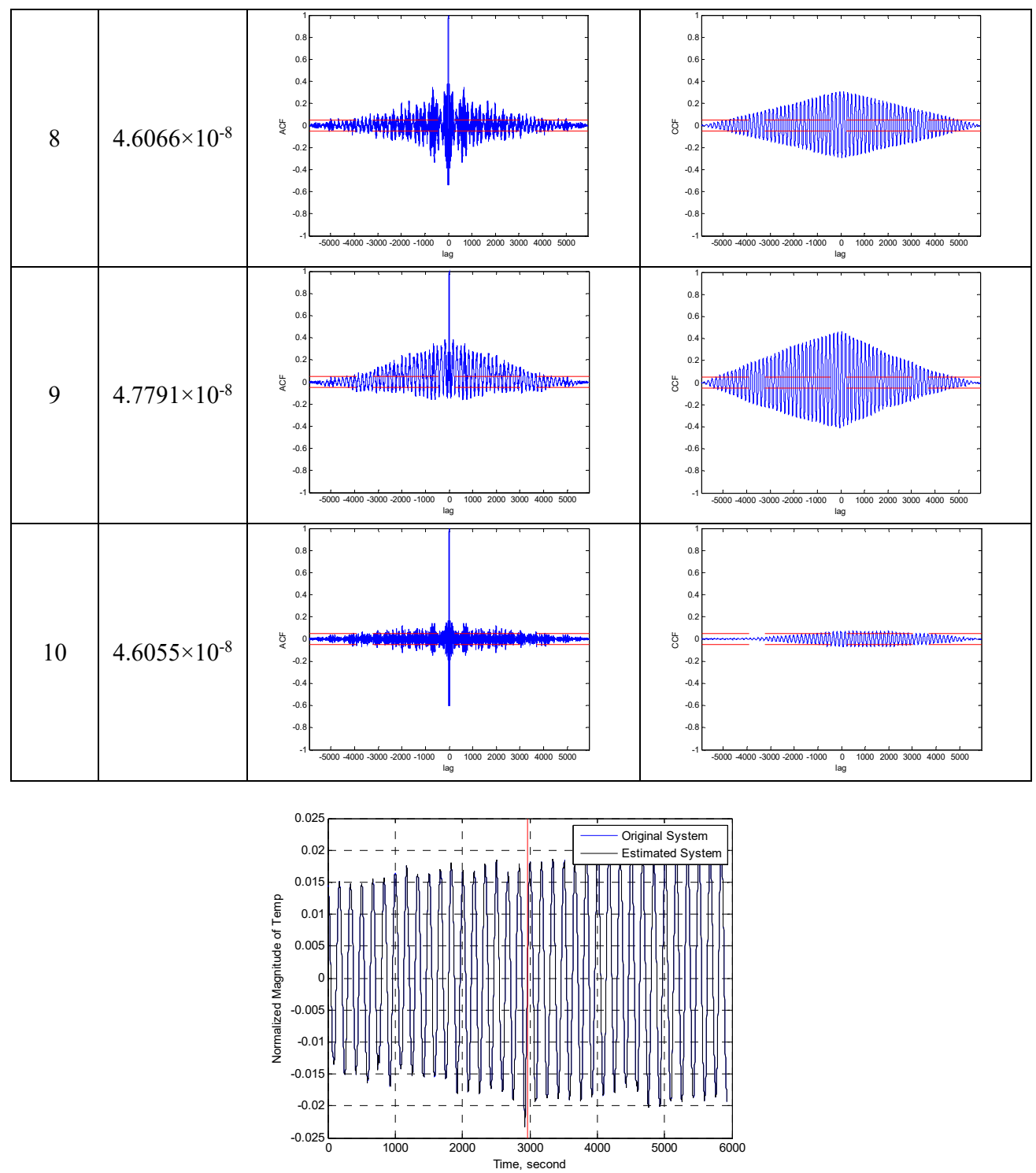

Fig. 19. The vibration of beam in time domain modeled by ABC algorithm

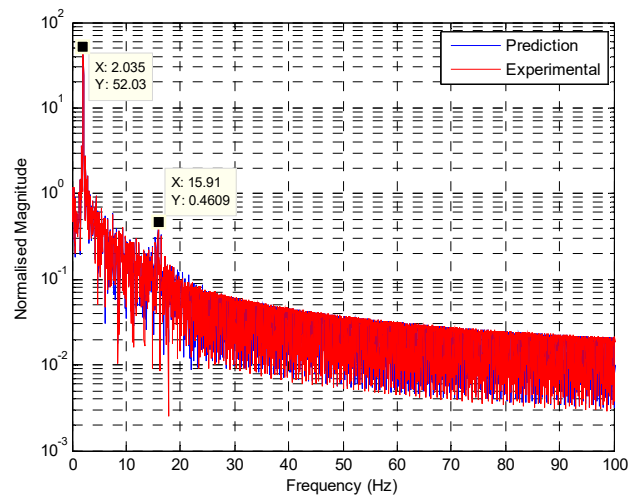

Fig. 20. The comparison of vibration modes between the predicted model and the real system 
Rickey Ting Pek Eek has conducted all the experiments and collect all the data collection. He contributed also in modelling the beam structure using Particle Swarm (PSO) algorithm. He also contributed in writing the paper. Intan Z. Mat Darus has also modelled the beam structure using $\mathrm{ABC}$ algorithm and conduct all the analysis of the performance. She also contributed in writing the paper. Shafishuhaza Sahlan has worked on the simulation and validation of the algorithms used in this paper. She also contributed in writing the paper. Pakharuddin Mohd Samin has worked on the design development and fabrication of the experimental setup. He supervised Mr. Rickey on experimental test and data collection. Dr Mohd Samin has also contributed on producing the experimental diagrams used in the paper. He also contributed in writing the paper. Nik M. R. Shaharuddin has worked on the integration of the experimental setup with data acquisition system. He also supervised Mr. Rickey on experimental test and data collection. Dr Shaharuddin has also contributed on producing the experimental diagrams used in the paper. He also contributed in writing the paper.

\section{Conclusion}

This paper proposed the optimization techniques which are applied in System Identification for modeling a flexible beam system. An experiment test rig for the beam system is built and developed to collect the data input and output experimentally for System Identification. Furthermore, a linear ARX model is estimated to be the model of the beam system. The optimization techniques are applied to estimate the unknown parameters in the ARX model. On top of that, several validation tests such as MSE and correlation tests are carried out to ensure the predicted model is close to the real system. In this paper, Particle Swarm Optimization and Artificial Bee Colony are the techniques used for parameters estimation of an ARX model.

From the results obtained by PSO technique, a 3rd order model is selected as the best fit model with the lowest MSE value of $1.9016 \times 10^{-10}$. Its correlation tests show that the PSO model is within the $95 \%$ confidence interval and close to the real system. Furthermore, in time domain, the predicted output is the same as the original output taken from experiment. Moreover, the natural frequencies for the first 2 vibration modes of the predicted model and original system are the same as well.

Meanwhile, it can be observed that $\mathrm{ABC}$ technique is able to develop a 2nd order model with a low MSE value of $2.1723 \times 10^{-9}$. In time domain, the predicted output is exactly the same as the original output taken from experiment. Apart from that, the natural frequencies for the first 2 vibration modes of the predicted model and original system are similar. However, based on the correlation tests, the behavior of $\mathrm{ABC}$ model is biased and less significant relationship between the input and residual compared to the $3 \mathrm{rd}$ order PSO model. Hence, the 2 nd order ABC model is unable to represent the real system of the flexible beam structure. On the contrary, the PSO model is the most appropriate and suitable as the predicted model for the flexible beam system as it consists of the lowest MSE and good correlation tests between the input and residual.

In the future work, the PSO model obtained will be validated through the real experiment by designing a controller to suppress the vibration of the beam system. The vibration must be reduced using this PSO model with the controller such as PID, direct controller, etc. Furthermore, Artificial Bee Colony is still a very new intelligence algorithm. It can be modified and improved for modeling in order to obtain an appropriate and accurate model of the beam system.

\section{Acknowledgements}

The authors wish to thank the Ministry of Higher Education (MOHE) and Universiti Teknologi Malaysia (UTM) for providing the research grants and facilities. This research is supported using Prototype Research Grant Scheme, Vote No. 4L661 and Research University Grant Vote No. 02K21. 


\section{References}

[1] Zadeh L. From circuit theory to system theory. Proceedings of the IRE, Vol. 50, Issue 5, 1962, p. 856-865.

[2] Eykhoff P. System Identification: Parameter and State Estimation. John Wiley and Sons, 1974, p. 555.

[3] Ljung L. Convergence analysis of parametric identification methods. IEEE Transactions on Automatic Control, Vol. 23, Issue 5, 1978, p. 770-783.

[4] Fu Li, Li Pengfei The research survey of system identification method. 5th International Conference on Intelligent Human-Machine Systems and Cybernetics, 2013.

[5] Xu Xiaoping, Wang Feng, Qian Fucai Study of Method of Nonlinear System Identification. 4th International Conference on Intelligent Computation Technology and Automation, 2011.

[6] Md Shariff Haslizamri, Hezri Mohd, Rahiman Fazalul, Tajjudin Maziadah Nonlinear system identification: comparison between PRMS and random gaussian perturbation on steam distillation pilot plant. IEEE 3rd International Conference on System Engineering and Technology, 2013.

[7] Md Lazin Md Norazlan, Mat Darus Intan Z., Chiang Ng Boon, Kamar Haslinda Mohamed Identification for automotive air-conditioning system using particle swarm optimization. Australian Control Conference, 2013.

[8] Sukri Hadi Muhammad, Mat Darus Intan Z., Yatim Hanim Modeling flexible plate structure system with free-free-clamped-clamped (FFCC) edges using particle swarm optimization. IEEE Symposium on Computers and Informatics, 2013.

[9] Yatim Hanim, Mat Darus Intan Z., Sukri Hadi Muhammad Particle swarm optimization of a flexible manipulator system. IEEE Symposium on Computers and Informatics, 2013.

[10] Li Wenbo, Wang Daqi, Liu Chengrui System identification of large flexible appendage on satellite for autonomous control. IEEE International Conference on Control and Automation (ICCA), 2013.

[11] Rodriguesz-Garxia Luis, Pérez-Londono Sandra, Mora-Florez Juan Particle swarm optimization applied in power system measurement-based load modeling. IEEE Congress on Evolutionary Computation, Cancun, Mexico, 2013.

[12] Saha S. K., Mandal D., Kar R., Saha Mallika IIR system identification using particle swarm optimization with improved inertia weight approach. 3rd International Conference on emerging Applications of Information Technology (EAIT), 2012.

[13] Deng Xiuqin System identification based on particle swarm optimization algorithm. International Conference on Computational Intelligence and Security, 2009.

[14] Dai Yuntao, Liu Liqiang, Song Jingyi Complex nonlinear system identification based on cellular particle swarm optimization. Proceedings of IEEE, International Conference on Mechatronics and Automation, Takamatsu, Japan, 2013.

[15] Mohamad M., Tokhi M. O., Toha S. F., Latiff I. Abd Particle swarm modelling of a flexible beam structure. 3rd UKSim European Symposium on Computer Modeling and Simulation, 2009.

[16] Gerhardt Eduardo, Martins Gomes Herbert Artificial bee colony (ABC) algorithm for engineering optimization problems. 3rd International Conference on Engineering Optimization EngOpt, 2012.

[17] Karaboga D. An Idea Based on Honeybee Swarm for Numerical Optimization. Technical Report TR06, Erciyes University, Engineering Centre, Cardiff University, UK, 2005.

[18] Jia Liu, Li Jing, Wang Junfeng, Feng Bo, Huo Junyi Research on the solving of nonlinear equation group based on artificial bee colony algorithm. 7th International Conference on Computer Science and Education, 2012.

[19] Ercin Ozden, Coban Ramazan Identification of linear dynamic systems using the artificial bee colony algorithm. Turkish Journal of Electrical Engineering and Computer Sciences, Vol. 20, 2012.

[20] Ravi V., Duraiswamy K. Artificial bee colony optimization for effective power system stabilization. Journal of Electrical and Electronics Engineering, Vol. 11, Issue 2, 2011.

[21] Karaboga D., Akay Bahriye Applied mathematics and computation. Applied Mathematics and Computation, Vol. 214, 2009, p. 108-132.

[22] Kennedy J., Eberhart R. C. Particle swarm optimization. IEEE Service Center, IEEE International Conference on Neural Network, Perth, Australia, Vol. 4, 1995, p. 1942-1948. 


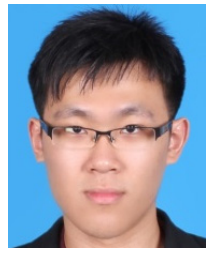

Rickey Ting Pek Eek was born in Sibu, Sarawak, in April 19th, 1988. He received his First Class Diploma in Electrical Electronics Engineering from Universiti Teknologi Malaysia (UTMKL), Kuala Lumpur in 2009 and later his First Class B.Eng. degree in Electrical Engineering of Control and Instrumentation from Universiti Teknologi Malaysia (UTMJB), Skudai Johor in 2012.Now he continues his study in Ph.D. in Mechanical Engineering of Applied Mechanics supervised by Assoc. Prof. Dr. Intan Z. Mat Darus and Dr. Shafishuhaza Sahlan. His current research interests are active vibration control and modeling of flexible beam structure, soft computing for swarm intelligence algorithms for system identification and control, and graphical user interface for simulation and experimental

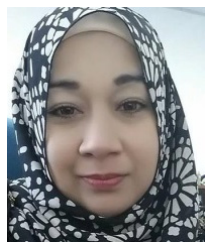

Intan Z. M. Darus was born in Melaka, Malaysia, in September 16th, 1976. She received her First Class B.Eng. (Hons.) degree in Mechanical Engineering from the University of Wales College Cardiff, Wales, United Kingdom in 1998 and later her Ph.D. in Automatic Control and Systems Engineering from the University of Sheffield, United Kingdom in 2004. Currently, she is an Associate Professor in the Department of Applied Mechanics and Design, Faculty of Mechanical Engineering, University Teknologi Malaysia. Her current research interests are active vibration control, modelling and simulation of dynamical system, soft computing and artificial intelligent techniques for system identification and control.

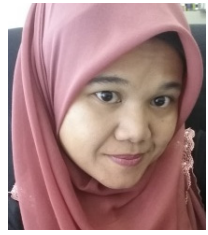

Shafishuhaza Sahlan was born in Selangor, Malaysia, in January 3rd, 1979. She received her M.Eng. (Hons.) degree in Control Systems Engineering from the University of Sheffield UK in 2002. Following that, she received her Ph.D. in Advancement in Control System Engineering from the university of Western Australia in 2010. Attached with the Faculty of Electrical Engineering in Universiti Teknologi Malaysia, Skudai, Johor, her research interest revolves around control system algorithm as well as process control and the industry. Currently, her research area of interest is on water treatment plant, focusing on power management, reduction of water wastage as well as a supervisory system of the plant.

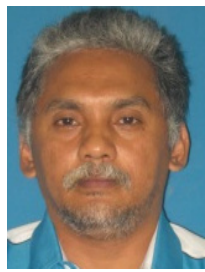

Pakharuddin Mohd Samin received both his B.Sc. and M.Sc. degrees from the Department of Mechanical Engineering, Texas A\&M University, USA; and received his Ph.D. in Mechanical Engineering from the Universiti Teknologi Malaysia. During 1995-1997, he worked at Bath University of UK on the design of an active roll control suspension system for passenger vehicle. He is currently an Associate Professor and head of department in the Department of Aeronautics, Automotive and Ocean Engineering, Faculty of Mechanical Engineering, Universiti Teknologi Malaysia. His research interests include continuously variable damper, ride and handling of vehicle and vehicle dynamics control.

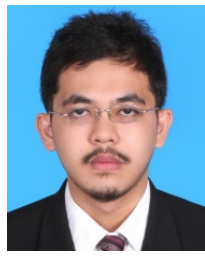

Nik Mohd Ridzuan Shaharuddin received his First Class B.Eng. in mechanical engineering (marine technology) in 2011 and his Ph.D. in mechanical engineering from the Universiti Teknologi Malaysia (UTM), Malaysia in 2015. During his Ph.D., he worked on the application of the active control on modelled segmented marine riser. Currently, he is in the Department of Aeronautics, Automotive and Ocean Engineering, Faculty of Mechanical, Universiti Teknologi Malaysia. His current research interests include active control, ship control system and vortex induced vibration. 